Recepción: 22 / 09 / 2016

Aceptación: 15 / 02 / 2017

Publicación: 29 / 04 / 2017

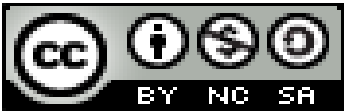

Ciencias de la educación

Artículo de investigación

\title{
Las técnicas de animación como instrumento de motivación para la enseñanza de la química y la biología
}

\author{
Animation techniques as an instrument of motivation for the teaching of \\ chemistry and biology
}

Técnicas de animação como ferramenta de motivação para ensino de química e biologia

\author{
Luzmila M. Valverde-Medina ${ }^{\mathrm{I}}$ \\ luzmilavalverde@yahoo.es \\ Luis C. Caicedo-Perlaza ${ }^{\text {II }}$ \\ luiscaicedoperlaza@yahoo.es
}

Correspondencia: luzmilavalverde@yahoo.es

\footnotetext{
I Profesor de Segunda Enseñanza Especialidad Químico-Biólogo, Licenciado en Ciencias de la Educación Especialidad Química y Biología, Diplomado Superior en Práctica Docente Universitaria, Especialista en Educación Superior, Magister en Investigación para el Desarrollo Educativo, Docente de la Universidad Luis Vargas Torres, Esmeraldas, Ecuador.

II Profesor de Segunda Enseñanza Especialidad Químico-Biólogo, Licenciado en Ciencias de la Educación Especialidad Química y Biología, Diplomado Superior en Práctica Docente Universitaria, Especialista en Educación Superior, Magister en Investigación para el Desarrollo Educativo, Docente de la Unidad Educativa San Daniel Comboni, Esmeraldas, Ecuador.
} 


\section{Resumen}

Con herramientas que proporciona la Animación Sociocultural como proceso, el docente puede aplicar estrategias que tributen a lograr adecuados niveles motivacionales en los estudiantes, sobre todo en las áreas de química y biología, por ser éstas dos de las disciplinas que más dificultades presentan en su enseñanza, buscando sobre todo lograr que los estudiantes sean los protagonistas de su propio aprendizaje. La definición más aceptada, más por el carácter universal del organismo que la propone que por la introducción de elementos que le impriman singularidad con respecto al de otros autores, es la aportada por la UNESCO, donde se plantea que: "La animación sociocultural es el conjunto de prácticas sociales que tienen por finalidad estimular la iniciativa y la participación de las comunidades en el proceso de su propio desarrollo y en la dinámica global de la vida sociopolítica en que están integradas. Las características de esta estrategia se pueden resumir en las siguientes: interdependencia positiva, interacción positiva, exigibilidad individual / responsabilidad personal, habilidades cooperativas para el funcionamiento efectivo del grupo, la toma de decisiones, saber generar confianza, autoanálisis del grupo. De esta manera, se procura una enseñanza dinámica y atractiva, en todo lo posible, práctica y experimental, según procedimientos que garanticen un proceso de enseñanza y siga la línea de filosofía constructivista.

Palabras clave: técnicas de animación; enseñanza; química; biología.

\section{Abstract}

With tools provided by Sociocultural Animation as a process, the teacher can apply strategies that contribute to achieve adequate levels of motivation in students, especially in the areas of chemistry and biology, as these are two of the disciplines that present more difficulties in teaching, seeking above all to make students the protagonists of their own learning. The most accepted definition, more because of the universal nature of the organization that proposes it, because of the introduction of elements that give it a singularity in relation to that of other authors, is that provided by UNESCO, which states that: "Socio-cultural animation is Set of social practices whose purpose is to stimulate the initiative and participation of the communities in the process of their own development and in the global dynamics of the socio-political life in which they are integrated. The characteristics of this strategy can be summarized as follows: positive interdependence, positive interaction, individual accountability / personal responsibility, 
cooperative skills for effective group functioning, decision making, confidence building, group self-analysis. In this way, a dynamic and attractive teaching is sought, in all possible, practical and experimental, according to procedures that guarantee a teaching process and follow the line of constructivist philosophy.

Keywords: animation techniques; teaching; chemistry; biology.

\section{Resumo}

Com as ferramentas fornecidas pelo Animação Sociocultural como um processo, os professores podem implementar estratégias que pagam impostos para atingir níveis de motivação adequados entre os alunos, especialmente nas áreas de química e biologia, porque estas são duas das disciplinas que a maioria das dificuldades apresentadas no seu ensino, olhando especialmente para garantir que os estudantes são os protagonistas de sua própria aprendizagem. A mais aceita mais pelo caráter universal do corpo que propõe a introdução de elementos que imprimem singularidade em relação a outros autores, é a prevista pela UNESCO, onde se afirma que a definição: "A animação sociocultural é o conjunto de práticas sociais que visam incentivar a iniciativa ea participação das comunidades no processo de seu próprio desenvolvimento e dinâmica global da vida sócio-política em que estão integrados. As características desta estratégia pode ser resumida da seguinte forma: a interdependência positiva, interação positiva, a exigibilidade responsabilidade individual / pessoal, habilidades cooperativas para funcionamento eficaz do grupo de tomada de decisão, ou seja, construir a confiança, grupo de auto-análise. Assim, uma instrução dinâmico e atraente busca, tanto quanto possível, prático e experimentais, de acordo com os procedimentos para assegurar um ensinamento e seguem a linha de filosofia construtiva.

Palavras chave: técnicas de animação; educação; química; biologia.

\section{Introducción}

La Animación Sociocultural, es concedida por diversas disciplinas del conocimiento científico de las áreas sociales y humanísticas para su fundamentación en torno a la Cultura principalmente en los estudios culturales de comunidades. La Animación Sociocultural es utilizada como una herramienta de cambio de patrones sociales y culturales principalmente por los métodos y 
técnicas que emplea, la cual constituye una metodología que ofrece alternativas para realizar estudios socioculturales y desarrollar procesos participativos en la comunidad.

Existen diversos autores que a partir de múltiples enfoques, han dedicado su análisis a la animación sociocultural, entre ellos se podrían mencionar, Pierre Besnard, Ezequiel Ander Egg, Toni Puig Picart, Rafaela Macías, José María Quintana, Ana Calvo y otros, cuyas definiciones y análisis sobre el tema coincide con la mayoría de la bibliografía referida al tema.

Si se parte de que la finalidad esencial de la Animación Sociocultural es estimular la iniciativa y la participación de los sujetos en su propio desarrollo, en el contexto educativo esta definición alcanza especial singularidad en su aplicación a la práctica. Al considerar la escuela como espacio comunitario, cuya dinámica se sostiene en la interacción entre docentes y estudiantes, la Animación Sociocultural puede convertirse es un instrumento de gran valía para lograr elevar la motivación de los estudiantes hacia el estudio. La relación existente entre el profesor y los alumnos puede depender de muchas circunstancias. De entre ellas, específicamente el uso de técnicas de animación sociocultural en el aula hace que se perfeccionen las relaciones interpersonales entre estos agentes educativos.

En el presente trabajo se pretende reflexionar acerca de cómo desde las herramientas que proporciona la Animación Sociocultural como proceso, el docente puede aplicar estrategias que tributen a lograr adecuados niveles motivacionales en los estudiantes, sobre todo en las áreas de química y biología, por ser éstas dos de las disciplinas que más dificultades presentan en su enseñanza, buscando sobre todo lograr que los estudiantes sean los protagonistas de su propio aprendizaje.

\section{Desarrollo}

Para entender la eficacia de la animación como instrumento didáctico dirigido a elevar la motivación en los estudiantes, hay que establecer los tipos de recursos técnicos, conceptuales y contextuales que en esta confluyen que son la base para una producción gráfica eficiente, sea animada o estática. 
La definición más aceptada, más por el carácter universal del organismo que la propone que por la introducción de elementos que le impriman singularidad con respecto al de otros autores, es la aportada por la UNESCO, donde se plantea que: "La animación sociocultural es el conjunto de prácticas sociales que tienen por finalidad estimular la iniciativa y la participación de las comunidades en el proceso de su propio desarrollo y en la dinámica global de la vida sociopolítica en que están integradas”.

Lo esencial de esta definición, ubicándola dentro del contexto educativo, es la importancia cada vez mayor que se le otorga al alumnado. Se debe lograr una sistemática interacción tanto con el profesor como con sus compañeros mostrándose más participativo e involucrado en el devenir de las clases de cara a construir su propio aprendizaje.

En general, los autores que tratan el tema, consideran que la animación sociocultural puede ser valorada desde diferentes puntos de vista y formas según la arista desde la cual se enfoque su estudio. Consideran factible concebirla atendiendo a dos de sus características: como un proceso encaminado a organizar y promover las acciones de las personas para gestar proyectos de desarrollo endógeno desde la cultura y para el desarrollo social; y como una metodología participativa dirigida a estimular la intervención de grupos en la interpretación y solución de problemas de carácter sociocultural de las comunidades.

Lo cierto es que, desde las múltiples concepciones y diferentes perspectivas en que se analiza la Animación Sociocultural, emergen dos aspectos comunes a todas: por una parte que la Animación Sociocultural se distingue por la manera de llevar a cabo las tareas concretas y las actividades específicas, y no por éstas en sí mismas; y por otra parte que es una técnica de intervención social en y con los grupos, que tiene por finalidad promover la cultura popular. A través de sus actividades la Animación Sociocultural trata de potenciar culturalmente a personas, grupos y comunidades, sin liderazgos y conducción exterior.

Al respecto Macías afirma que la Animación Sociocultural es una alternativa para promover la participación efectiva y concreta de la comunidad o grupo social en la fijación de prioridades, la toma de decisiones y la elaboración y puesta en marcha de estrategias de planificación para alcanzar un mejor nivel en sentido general. La fuerza motriz de este proceso proviene del poder 
real de las comunidades, de la posesión y del control que tengan sobre sus propios empeños y destinos. El desarrollo de la comunidad se basa en los recursos humanos y materiales con que cuenta la comunidad misma para estimular la independencia y el apoyo social, así como para desarrollar sistemas flexibles que refuercen la participación pública y el control de las cuestiones culturales, esto requiere un total y constante acceso a la información de su contexto (Macías, 2000: 42).

Ander Egg sitúa las características principales de la Animación Sociocultural como: un proceso participativo que trabaja desde el universo complejo y diverso de la Cultura, en su amplia expresión, acciona a la vez con la memoria histórica; fortalece y reafirma la Identidad Cultural. Como proceso participativo sustenta su intervención en conocimientos teóricos y métodos de las Ciencias Sociales y Humanísticas. Implica accionar con propuestas metodológicas dirigidas a realizar análisis, programación, ejecución y evaluación de acciones que faciliten y potencian las transformaciones en grupos y comunidades (Ander, 2005).

Los métodos y técnicas con los que opera, permiten lograr la real participación de la población desde los mismos inicios del proceso, lo que garantiza el carácter endógeno de las propuestas de cambios que también generan su utilización.

El hecho de que la Animación Sociocultural trabaja en, con y para grupos brinda además, propuestas para consolidar lo mejor de cada situación específica. Aporta metodologías, programas, proyectos, sistemas de acciones, modelos, todos instrumentos que favorecen la gestión. Provee a los que la asumen, un cuerpo teórico argumentado, necesario para conducir con acierto los complejos procesos insertos en la dinámica global social.

La Animación Sociocultural aspira a lograr la formación integral de las personas y a mejorar su calidad de vida, y contrapone el logro de una verdadera democracia cultural a la simple extensión o democratización de la cultura, con el fin de promover la emancipación colectiva y el cambio social.

La animación sociocultural como herramienta para el contexto educativo puede convertirse en un mecanismo que contribuya a estimular la creatividad, participación, cohesión grupal y la 
satisfacción de las necesidades reales de los educandos. Cuenta con una serie de recursos que son eficaces para, mediante la realización de acciones determinadas, llevar a cabo un proceso global que contribuya al fortalecimiento de las capacidades y el desarrollo de habilidades en los estudiantes.

La animación sociocultural se vuelve una alternativa para elevar la motivación al potenciar el trabajo grupal mediante proyectos en los cuales se implique a todos los factores y miembros que la integran.

Como una de las alternativas que ofrece la Animación Sociocultural para la enseñanza, Manuel Castells (1986) propone el lenguaje audiovisual desde lo comunicacional a través del uso de las TIC en cuanto hacen parte de una nueva base que promociona la educación pública de manera que "comprenda una serie de aplicaciones de descubrimiento científico cuyo núcleo central consiste en una capacidad cada vez mayor de tratamiento de la información" (Castells, 1986; 34).

Siguiendo esta misma línea, Bonafé Martínez (2008) cuestiona el libro de texto como una herramienta que homogeniza el desarrollo curricular en un sistema educativo escolástico cerrado, impidiendo que nuevos modelos pedagógicos alternativos, en esta época de la información, la comunicación y la inmediatez tecnológica sean aprovechados. Concibe a las Tecnologías de la Información y Comunicación (TIC) como elementos que ofrecen bondades y conveniencias para la aprehensión de nuevos conocimientos permitiendo al educando vivenciar el uso real del conocimiento fuera del aula a través de diferentes dispositivos o medios.

Existe una serie de principios de percepción y recepción sensorial que activan estímulos en la mente humana. Según como se activen, se puede generar recordación y fácil lectura de los mensajes. Uno de los principios fundamentales parte de la corriente Gestalt y es el llamado Principio de la Pregnancia, que afirma la tendencia de la experiencia perceptiva a adoptar las formas más simples posibles. En consecuencia se facilita la lectura de mensajes al desglosar su significado evitando que interfiera con su propósito comunicacional. (Perls, f, Hefferline, r. \& Goodman, P. 1965). 
Perls, Hefferline y Goodman proponen también otros principios. Entre ellos se destacan el Principio de la Semejanza, donde el observador tiende a agrupar los elementos similares en la forma, el tamaño, el color y otros aspectos visuales. Principio de la Proximidad: proponiendo que a la menor distancia, se favorece el agrupamiento parcial o secuencial de elementos. Principio de Simetría: las imágenes simétricas son percibidas como un solo elemento en la distancia. Estos principios de percepción son base para la biología, la matemática, la química, la física y hasta la misma estética, ya que se organizan siguiendo las leyes especulares, simples o múltiples, de la simetría. Finalmente, el Principio de Continuidad: el observador percibe elementos continuos aunque estén interrumpidos entre sí, como parte de un modelo.

En otro orden, es necesario destacar además la fuerte tendencia del uso de recursos lúdicos en las actividades de enseñanza- aprendizaje para facilitar la adquisición de conocimientos nuevos en todos los niveles educativos y de manera muy importante en la enseñanza de ciencias como la química y la biología.

En dichas áreas del conocimiento según Garritz, Rueda, Robles y Vázquez (2011), se deben desarrollar sujetos autodidactas que apliquen el método científico y tengan conciencia del impacto de la ciencia y la tecnología sobre la sociedad y su entorno.

Para Orozco (2014) el aprendizaje basado en juegos permite integrar estrategias didácticas para mejorar el desarrollo de habilidades; además agrega que los juegos digitales son cada vez más utilizados en el área educativa. Lo anterior está fundamentado en la premisa de que por este medio, el alumno se convierte en un ente activo y se involucra completamente en el proceso de enseñanza-aprendizaje.

En la enseñanza de las ciencias, el experimento desempeña un papel importante, ya que, también de despertar el interés por el aprendizaje y de crear incentivos para mejorar la asimilación del contenido, permite el trabajo colectivo y práctico como fuente de adquisición de los conocimientos y también contribuye a que se aprenda a ver en la práctica la confirmación de las teorías y postulados científicos. Esto sustenta la necesidad de aplicar las herramientas que proporciona la Animación Sociocultural en el proceso de enseñanza aprendizaje. 
Un balance de materiales conveniente para una formación química requiere, desde el comienzo, un aprendizaje experimental, personal y directo, de los tipos más representativos de técnicas y métodos. Esa mezcla de teoría y práctica debe ser equitativa en cuanto a la capacidad material para asimilar los conocimientos. Por consiguiente, se debe buscar equilibrar la enseñanza teórica con más horas de laboratorio para conseguir, de esta forma, una mayor coherencia en los temas que requieren de la experimentación.

La química enseñada con propiedad permite una de las más elevadas conquistas de la condición humana, ya que el aprendiz puede realizar, en forma individual, lo planeado o previsto, claro está, después de estudios teóricos y de razonamientos lógicos, de manera que armonice el trabajo manual con el pensamiento intelectual. De aquí lo significativo de la existencia de técnicas lúdicas o de animación que tributen no sólo a impartir con eficiencia la enseñanza de la química sino a lograr el desarrollo del autoaprendizaje en los estudiantes.

Con respecto a la enseñanza de la biología están siendo cada vez más frecuentes determinadas propuestas imaginativas del profesorado, que persiguen una enseñanza motivadora de la Biología e intentan dar respuesta a las expectativas que se esperan de su aprendizaje. Para ello los docentes han recurrido a distintas fórmulas de perfeccionamiento: en unos casos a una especialización didáctica en la formación o, en otros, a la formación a través de acciones formativas puntuales, grupos de trabajo o proyectos de innovación educativa.

Como parte de este proceso se ha aprovechado el importante papel de la Biología en la Educación en Valores Positivos para el ser humano y en las enseñanzas transversales como Educación para el Desarrollo Sostenible (EDS) o Educación para la Salud (ES). También la repercusión en la práctica diaria de tendencias como la Investigación-Acción, frecuentemente relacionadas con el tratamiento de la transversalidad.

Una de las principales herramientas que ofrece la Animación Sociocultural para el ambiente educativo es el aprendizaje cooperativo. Este se ha definido como "pequeños grupos de personas que trabajan juntos como un equipo para resolver un problema, realizar una tarea o llegar a una meta común” (Artz y Newman, 1990, p. 448). Los estudiantes deben colaborar unos con otros 
para llegar a esas metas. No obstante, para llegar a esta interdependencia positiva es necesario un proceso planificado y pensado.

Esta estrategia se puede realizar de formas diversas dando unos resultados generales de aprendizaje satisfactorios. Las características de esta estrategia según Bará, Domingo y Valero (2007) se pueden resumir en las siguientes:

1. Interdependencia positiva: un estudiante piensa que no puede tener éxito si los restantes componentes del grupo no lo logran y viceversa.

2. Interacción positiva: los estudiantes se ayudan mutuamente explicándose los contenidos o problemas unos a otros.

3. Exigibilidad individual / responsabilidad personal: el profesor debe asegurarse de que se evalúen los resultados de cada estudiante.

4. Habilidades cooperativas para el funcionamiento efectivo del grupo: capacidades como el liderazgo, la toma de decisiones, saber generar confianza.

5. Autoanálisis del grupo: discusión dentro del grupo para saber en qué grado se están logrando los objetivos.

Algunas de las experiencias que se desarrollan como parte de este procedimiento en la enseñanza de la biología se desarrollan a través de comparaciones en el campo motivacional de una estrategia de aprendizaje cooperativo como el Jigsaw y el modelo de instrucción directa. Se cuenta con recientes investigaciones que han estudiado la eficiencia de diferentes metodologías de enseñanza en las que se visualiza que el efecto de la colaboración entre los alumnos a la hora de resolver situaciones específicas en el área de la biología, la aplicación del aprendizaje cooperativo con la ayuda de las nuevas tecnologías, el estudio de la eficiencia del aprendizaje cooperativo para la realización de algunas prácticas de laboratorio, en las que se analizan muestras de organismos, etc. 
Además, con esta estrategia se trabajan otros aspectos de capital importancia referidos a las actitudes de solidaridad, igualdad, respeto, diálogo y libertad. En consecuencia, el aprendizaje cooperativo producirá también un cambio positivo en la motivación.

\section{Conclusiones}

Existe una preocupación creciente en la labor docente por aplicar los métodos activos o actividades experimentales que despierten el interés del estudiantado; por lo que se debe adecuar el tratamiento pedagógico a las modalidades, aptitudes e intereses de los alumnos y alumnas. De esta manera, se procura una enseñanza dinámica y atractiva, en todo lo posible, práctica y experimental, según procedimientos que garanticen un proceso de enseñanza y siga la línea de filosofía constructivista.

\section{Referencias Bibliográficas}

Ander Egg, Ezequiel (2005): “Metodología y práctica del desarrollo de la comunidad”. Editorial Humanitas, Buenos Aires.

Castells, Manuel. (1986). El desafío tecnológico. España y las nuevas tecnologías, Madrid: Alianza Editorial.

Garritz, A., Rueda, C., Robles, C. y Vázquez, Á. A. (2011). Actitudes sobre la naturaleza de ciencia y tecnología en profesores y estudiantes mexicanos del bachillerato y la universidad públicos. Proyecto Iberoamericano de Evaluación de Actitudes Relacionadas con Ciencia, Tecnología y Sociedad. Educ. Quím., 22(2), 141---154.

Revista UNESCO. (2006) El patrimonio mundial, balances y perspectivas. Septiembre.

Macías Reyes, Rafaela (2000); “Compilación: Teoría de la cultura”, Santiago de Cuba.

Martínez Bonafé, J. (2008). Los libros de texto como práctica discursiva, Revista de la Asociación de Sociología de la Educación Vol 1.p. 65. Revistas en Línea. [Consultado 3 May 2016]. Disponible en: http://dialnet.unirioja.es/servlet/autor?codigo=114061 
Orozco, L. (2014). Estudio comparativo de los modelos de evaluación de la calidad e-learning en el Sistema de Universidad Virtual de la Universidad de Guadalajara México y propuesta complementaria. Tesis doctoral. [Consultado 3 May 2016]. Disponible en: http://www.tdx.cat/handle/10803/285341.

Perls, f, Hefferline, r. \&Goodman, P. (1965). Gestalt therapy: Excitement and growth in the human personality. New York: Julian Press. 